\title{
A noninvasive multi-analyte diagnostic assay: combining protein and DNA markers to stratify bladder cancer patients
}

This article was published in the following Dove Press journal:

Research and Reports in Urology

3 March 2012

Number of times this article has been viewed

\section{Cecilia A Fernandez' \\ John M Millholland' \\ Ellen C Zwarthoff ${ }^{2}$ \\ Adam S Feldman ${ }^{3}$ \\ $R$ Jeffrey Karnes ${ }^{4}$ \\ Anthony P Shuber'}

'Predictive Biosciences, Inc, Lexington, MA, USA; ${ }^{2}$ Erasmus Medical Center, Rotterdam, The Netherlands; ${ }^{3}$ Massachusetts General Hospital, Boston, MA, ${ }^{4}$ Mayo Clinic, Rochester, MN, USA
Correspondence: Anthony P Shuber Predictive Biosciences, 128 Spring Street, Lexington, MA 0242I, USA

Tel + I 78 I402 I780

Fax + I 78 |402 I785

Email tshuber@predictivebiosci.com
Purpose: The authors recently reported the development of a noninvasive diagnostic assay using urinary matrix metalloproteinases (MMPs) as monitors of disease-free status and bladder cancer in high-risk populations. Using an approach called clinical intervention determining diagnostic (CIDD), they identified with high confidence those patients who could be excluded from additional intervention. To maximize performance, MMPs were combined with DNAbased markers and CIDD was applied to a population of patients undergoing monitoring for recurrence.

Patients and methods: Urine samples were obtained from 323 patients, 48 of whom had a recurrence and 275 of whom did not have cancer upon cytoscopic evaluation. Twist1 and Nid2 methylation status was determined using methylation-specific polymerase chain reaction, FGFR3 mutational status by quantitative PCR, and MMP levels by enzyme-linked immunosorbent assay.

Results: Using a combination of these DNA and protein markers, the authors identified with high confidence ( $97 \%$ negative predicted value) those patients who do not have cancer. Cutoffs were adjusted such that at $92 \%$ sensitivity, $51 \%$ of disease-free patients might be triaged from receiving further tests.

Conclusion: The multi-analyte diagnostic readout assay described here is the first to combine protein and DNA biomarkers into one assay for optimal clinical performance. Using this approach, the detection of FGFR3 mutations and Twist1 and Nid2 methylation in the urine of patients undergoing bladder cancer recurrence screening increase the sensitivity and negative predictive value at an established MMP protein cutoff. This noninvasive urinary diagnostic assay could lead to the more efficient triage of patients undergoing recurrence monitoring.

Keywords: matrix metalloproteinase, FGFR3, bladder cancer, diagnostics

\section{Introduction}

In 2007, over 67,000 new cases of bladder cancer were diagnosed in the USA. ${ }^{1}$ Bladder cancer is the most costly of all cancers per patient, with average total costs ranging from $\$ 69,287$ to $\$ 202,203$. $^{2}$ Biomarkers with high specificity and sensitivity have the potential to significantly reduce costs, particularly because the prevalence of cancer in a population of patients undergoing routine evaluation for recurrence is low at any monitoring interval. To date, most noninvasive diagnostic assays are based upon statistical averaging over a large number of patients in order to arrive at thresholds that maximize sensitivity and specificity. In practice, performance characteristics of assays utilizing sensitivity and specificity allow a physician to determine a patient's relative risk with a certain degree of statistical confidence, but these assays can create ambiguity 
as to the desired course of management. Currently available biomarkers suffer from both low sensitivity and low specificity, particularly for low-grade tumors. ${ }^{3}$ This limitation is of importance when utilizing biomarkers for triaging highrisk populations such as bladder cancer survivors where single markers are unlikely to provide clinically relevant results. ${ }^{4}$ There is a clear need for novel clinical management approaches and assays that result in more efficient screening and management of high-risk patients.

Matrix metalloproteinases (MMPs) are a family of zincdependent endopeptidases that have been shown to be key regulators of tumor growth, angiogenesis, and metastasis formation. ${ }^{5,6}$ Increased MMP expression is required for tumors to grow into the surrounding tissue and for the dissemination of metastatic cells into the vasculature and distant sites. Detection of MMPs in the urine of cancer patients has been shown to correlate with disease status in a variety of cancers, ${ }^{7-11}$ including bladder cancer. ${ }^{7,9,12}$ This study and others like it have shown that biologically active MMP-2 and MMP-9 are found at higher levels and at greater frequency in the urine of cancer patients than in that of asymptomatic healthy controls.

In a recent study, the authors reported the development of a noninvasive diagnostic assay utilizing urinary MMPs as monitors of disease-free status and cancer in high-risk bladder cancer populations. ${ }^{12}$ The report outlined the use of urinary MMPs (either MMP-2 or MMP-9) as triage monitors to manage the care of high-risk populations by identifying with high confidence those patients who do not have bladder cancer. In their previous report, the authors described a clinical approach based on high negative predictive value (NPV), termed the clinical intervention determining diagnostic (CIDD), that can reduce ambiguity by identifying patients who do not have bladder cancer, stratifying the population into patients who should receive standard-of-care monitoring and patients who could potentially forgo cystoscopy. ${ }^{12}$

To complement their existing protein-based assay and increase performance in high-risk populations, the authors have developed noninvasive DNA-based assays for the detection of FGFR3 mutations and Twist1/Nid2 methylation in urine. Mutations of the FGFR3 gene have been observed in both bladder and cervical neoplasias. ${ }^{13}$ FGFR3 is a member of a family of four structurally related tyrosine kinase glycoprotein receptors encoded by separate genes. ${ }^{14}$ FGFR3 mutations in bladder tumors have been characterized in exon 7 , exon 10, and exon 15, and show a significant association with low-grade, noninvasive tumors. ${ }^{15-18}$

While FGFR3 mutations occur primarily in low-grade noninvasive tumors and MMPs are associated with higher-grade invasive tumors, methylation of Twist1 and Nid2 is not associated with tumor stage. Twist1 and Nid2 have been frequently shown to be methylated in various cancers including bladder cancer. ${ }^{19-22}$ A recent study has also shown that, together, the methylation status of Twist1 and Nid 2 can be used as independent predictors of bladder cancer in urine samples. ${ }^{23}$

The addition of DNA markers to the existing protein-based assay results in superior performance. DNA markers, which tend to have high specificity, increase sensitivity without much loss of specificity. This allows the protein marker cutoffs to be set at higher levels, increasing specificity without loss of sensitivity and NPV.

Using these markers and the CIDD concept, patients with MMP levels below the cutoffs could be potentially excluded from further intervention. The assay described here combines MMP-2, FGFR3 mutation detection and Twist1/Nid2 methylation analysis in one noninvasive test. This multi-analyte diagnostic readout (MADR) assay allows for the more efficient management of high-risk bladder cancer populations by potentially reducing the number of negative cystoscopies.

\section{Materials and methods Experimental samples}

Urine samples were collected from various academic institutions and urology practices. All sites participating received Institutional Review Board approval. Subjects were required to sign institutional review board-approved informed consents, prior to any study-related procedures being performed. All patients had previously been treated for bladder cancer and were undergoing routine monitoring for recurrence. Urine samples were collected using a standard clean catch protocol from 323 patients, including 48 patients identified by various means to have a bladder cancer recurrence (all tumors confirmed by pathology) and 275 patients who had no evidence of disease at the given monitoring interval. Table 1 describes the clinical characteristics of the test populations including stage and grade of the cancer samples. Urine samples were aliquoted and stored at $-80^{\circ} \mathrm{C}$ until assayed. For DNA analyses, urine samples were stabilized with $25 \mathrm{mM}$ EDTA prior to aliquoting and freezing.

\section{MMP-2 and MMP-9 ELISA}

To determine total MMP-2 and MMP-9 levels, $50 \mathrm{~mL}$ or $100 \mathrm{~mL}$ of neat urine, respectively, were processed through ELISAs specific for MMP-2 or MMP-9, as per the manufacturer's instructions (R\&D Systems, Minneapolis, MN). 
Table I The clinical characteristics of the test populations

\begin{tabular}{ll}
\hline Controls & Number of patients \\
\hline NED & 275 \\
Cancers & 48 \\
Stage & \\
Ta & 31 \\
Tis & 4 \\
T1 & 9 \\
T2 & 2 \\
T3 & 2 \\
Grade & \\
I & 26 \\
2 & 5 \\
3 & 17 \\
\hline
\end{tabular}

Abbreviation: NED, no evidence of disease.

All assays were performed blinded as to the clinical status of the samples.

\section{DNA isolation from urine}

DNA isolation was performed from whole urine to purify both cell-associated and free DNA. Urine aliquots were thawed at room temperature, and genomic DNA isolation was performed using the QIAamp Minelute Virus Vacuum Kit according to manufacturer's instructions. Purified DNA was eluted in $150 \mathrm{ul}$ AVE elution buffer or water and stored at $-20^{\circ} \mathrm{C}$ until ready to assay.

\section{FGFR3 exon-specific amplification (primary PCR)}

Primary PCR of genomic DNA extracted from $4 \mathrm{~mL}$ of urine was carried out using oligonucleotide primers specific for human FGFR3 to amplify DNA from exons 7, 10, and 15 (Table 2).

PCR amplification was performed using a C1000 thermal cycler (Bio-Rad Laboratories, Hercules, CA) under standard conditions. DNA amplification was confirmed by agarose gel analysis of primary PCR products.

\section{FGFR3 mutation detection}

FGFR3 mutations were detected by utilizing a PCR-clamping methodology. Wild-type blocking oligonucleotides containing locked nucleic acid (LNA) bases surrounding known mutation

Table 2

\begin{tabular}{|c|c|c|}
\hline Assay & Reagent & Sequence \\
\hline \multirow{6}{*}{$\begin{array}{l}\text { FGFR3 } \\
\text { primary PCR }\end{array}$} & Exon 7 forward & $5^{\prime}$ GCG GTC CCA AAA GGG TCA GTA CAG TGG CGG TGG TGG TGA GGG AG 3' \\
\hline & Exon 7 reverse & 5' GCG GTC CCA AAA GGG TCA GTA CGC ACC GCC GTC TGG TTG G 3' \\
\hline & Exon 10 forward & 5' GCG GTC CCA AAA GGG TCA GTA CGG TCT GGC CCT CTA GAC TCA 3' \\
\hline & Exon 10 reverse & 5' GCG GTC CCA AAA GGG TCA GTA CGG TCT GGC CCT CTA GAC TCA 3' \\
\hline & Exon 15 forward & 5' GCG GTC CCA AAA GGG TCA GTA CCC TGC CCT GAG ATG CT 3' \\
\hline & Exon 15 reverse & 5' GCG GTC CCA AAA GGG TCA GTA CCG TCC TAC TGG CAT GAC C $3^{\prime}$ \\
\hline \multirow{14}{*}{$\begin{array}{l}\text { FGFR3 mutation } \\
\text { detection }\end{array}$} & Exon 7 forward & $5^{\prime}$ GCG TCA TCT GCC CCC A 3' \\
\hline & Exon 7 reverse & $5^{\prime}$ CAC CGC CGT CTG GTT G $3^{\prime}$ \\
\hline & Exon 7 LNA & $5^{\prime}$ AGA GCG CTC CCC G $3^{\prime}$ \\
\hline & Exon 7 probe & 5' FAM-CCC GCC TGC AGG ATG GGC CGG T-lowa black FQ 3' \\
\hline & Exon 10 forward & 5' GGC CTC AAC GCC CAT GT 3' \\
\hline & Exon $10 \mathrm{~A}$ reverse & 5' TAG CTG AGG ATG CCT GCA TA 3' \\
\hline & Exon $10 \mathrm{~B}$ reverse & $5^{\prime}$ CCG TAG CTG AGG ATG CCT G 3' \\
\hline & Exon IOA LNA & $5^{\prime}$ ATA CAC ACT GCC CGC CT 3' \\
\hline & Exon IOB LNA & $5^{\prime}$ GCC TGC ATA CAC ACT 3' \\
\hline & Exon 10 probe & 5' FAM-CCG AGG AGG AGC TGG TGG AGG CTG AC-lowa black FQ 3' \\
\hline & Exon 15 forward & 5' CAA TGT GCT GGT GAC CGA G 3' \\
\hline & Exon 15 reverse & $5^{\prime}$ CCG GGC TCA CGT TGG TC $3^{\prime}$ \\
\hline & Exon I5 LNA & 5' GGT CGT CTT CTT GTA GT 3' \\
\hline & Exon 15 probe & 5' FAM-CTG GCC CGG GAC GTG CAC AAC CTC GAC T-lowa black FQ 3' \\
\hline \multirow[t]{4}{*}{ Twist/Nid } & Twist forward & 5' GTT AGG GTT CGG GGG CGT TGT T 3' \\
\hline & Twist reverse & $5^{\prime}$ CCG TCG CCT TCC TCC GAC GAA 3' \\
\hline & Nid forward & 5' GCG GTT TTT AAG GAG TTT TAT TTT C 3' \\
\hline & Nid reverse & 5' CTA CGA AAT TCC CTT TAC GCT 3' \\
\hline \multirow[t]{3}{*}{ ACTB } & ACTB forward & $5^{\prime}$ TAG GGA GTA TAT AGG TTG GGG AAG TT $3^{\prime}$ \\
\hline & ACTB reverse & $5^{\prime}$ AAC ACA CAA TAA CAA ACA CAA ATT CAC $3^{\prime}$ \\
\hline & ACTB zen probe & 5’ TGG GGT GGT/ZEN/GAT GGA GGA GGT TTA GTA AGT TTT TT 3' \\
\hline
\end{tabular}

Abbreviations: ACTB, Actin- $\beta$; PCR, polymerase chain reaction. 
sites were included along with real-time PCR primers and dual-labeled Taqman probes. Real-time PCR amplification was performed using a Light Cycler real-time thermal cycler (Roche Diagnostics Corporation, Indianapolis, IN). Dual realtime PCR reactions, with and without the LNA blocker, were assembled in duplicate for each amplification. Positive control plasmids were designed to incorporate the region of the mutation, as well as flanking sequences to facilitate amplification of the positive control plasmid during the primary PCR step described above. Each positive control plasmid contains a single nucleotide change, confirmed by sequencing.

\section{Twist l/Nid2 methylation detection}

Detection of methylated Twist 1 and Nid2 was carried out by conventional methylation specific PCR. For this assay, DNA from $8 \mathrm{~mL}$ of urine was extracted as described above and eluted in water. DNA yield was determined by quantitative real-time PCR using a reference gene. A maximum of $2 \mathrm{ug}$ of DNA was concentrated using AES 1000 Speed Vac (Thermo Fisher, Waltham, MA). Each sample was resuspended in water by pipetting. Bisulfite conversion of the DNA was performed using a Qiagen Epitect Bisulfite Kit following the manufacturer's directions. The resulting converted DNA was loaded on columns, subjected to desulfonation, washed, and eluted in $30 \mu \mathrm{L}$ of molecular grade water and placed at $-20^{\circ} \mathrm{C}$ until assayed.

Conventional MSP was performed using methylationspecific primers to sequences within the promoter region of Twist1 and Nid2. The primer sequences are as listed in Table 2.

PCR amplifications were performed using the C1000 thermocycler under standard conditions. Each set of amplifications contained a water blank, unconverted human genomic DNA, 100\% methylated DNA, 100\% unmethylated DNA, and a $1 \%$ methylated DNA control.

In addition, to verify that negative samples had the required minimum of $10 \mathrm{ng}$ DNA content, $5 \mu \mathrm{L}$ of bisulfiteconverted DNA was quantitated by qPCR using primers specific for a nonmethylated region of Actin-B (Table 2). Actin-B quantitation was determined using a standard curve of unmethylated DNA (Qiagen), ranging from 0.4 to $50 \mathrm{ng}$ total input. Real-time PCR amplifications were carried out with the Roche LightCycler 480 under standard conditions.

\section{Biomarker performance analysis using MADR}

Samples positive for FGFR3 were assigned a score of "1," while those negative for an FGFR3 mutation were assigned a score of " 0 ." For quantitative markers, individual marker cutoffs were established to maximize specificity. Each marker was then scored as " 1 " for above the cutoff or " 0 " for below the cutoff. The sum of all markers was used to establish final clinical performance. By assigning an individual score for each marker, DNA and protein markers are easily combined without the need of complex multivariate analyses. To obtain maximum negative predictive value, samples are considered negative when a total score of " 0 " is obtained. Patients with scores of " 0 " could be excluded from further testing with very high NPV. Samples with score of $\geq$ " 1 " are considered intermediate and, based on the CIDD concept, should remain in cue for standard of care. Final clinical performance of sensitivity, specificity, and NPV were calculated using standard methods. Confidence intervals were calculated using an excel macro binomial confidence interval calculator.

\section{Results \\ MMP-2 detection in urine and performance using CIDD}

The CIDD approach uses quantitative markers to triage patient populations. Using this approach, marker cutoffs are set to result in maximum NPV and sensitivity, such that patients who do not have cancer might be excluded from further intervention (Figure 1A). Patients could also have been triaged into those that might benefit from maximum intervention (high positive predictive value).

In the authors' previous study, they described the use of MMPs as bladder cancer markers in a population consisting of bladder cancer patients and asymptomatic controls. ${ }^{12}$ In this study, they determined MMP levels in a bladder cancer recurrence population using MMP specific ELISAs. In this clinical population, MMP-2 resulted in better clinical performance than MMP-9 (data not shown) and was therefore used in the MADR assay. As in previous studies, mean MMP-2 levels are higher in patients with cancer than in those with no evidence of disease $(1.337 \mathrm{ng} / \mathrm{mL}$ and $0.844 \mathrm{ng} / \mathrm{mL}$, respectively). However, in this high-risk patient group, the distribution of cancer and cancer-free patients is not as distinct, such that in order to achieve high NPV ( $\geq 90 \%$ ) with high sensitivity ( $\geq 90 \%$ ), protein cutoff values would have to be set lower than in asymptomatic populations (Figure 1B). In a recurrence monitoring population, MMP-2 cutoffs that result in $\geq 90 \% \mathrm{NPV}$ and sensitivity result in only $19 \%$ of the cancer-free population being excluded from further intervention. 
A

Asymptomatic Cancer

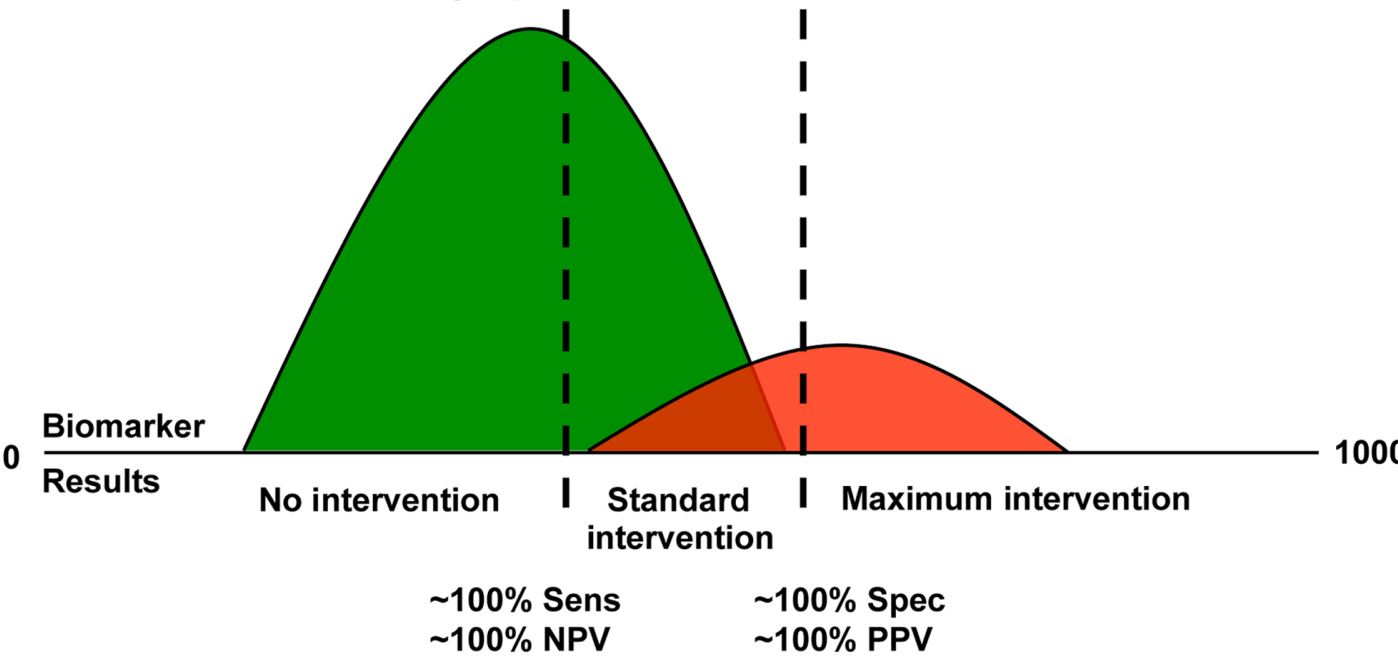

B

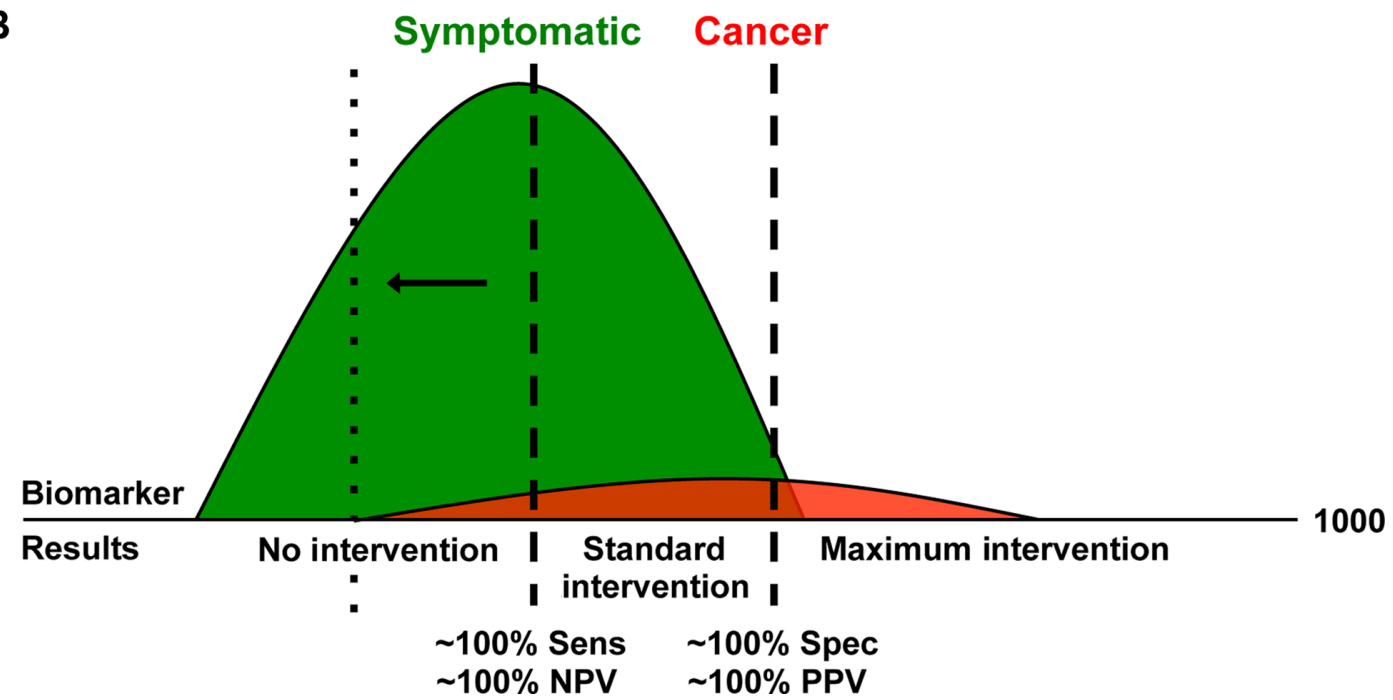

Figure I Clinical intervention determining diagnostic (CIDD) approach using matrix metalloproteinases (MMPs). (A) The CIDD approach uses one MMP cutoff to maximize sensitivity (Sens) and negative predictive value (NPV), and a second cutoff to maximize specificity (Spec) and positive predictive value (PPV). Patients with cutoff values in between these cutoffs continue to receive standard of care. (B) In high-risk populations, the MMP levels in the cancer and cancer-free populations have greater overlap, such that MMP cutoffs would have to be lowered to retain high NPV and sensitivity.

Abbreviations: PPV, positive predictive value; NPV, negative predictive value.

\section{MADR: combining protein and DNA markers}

Although protein marker levels can be set to maximize sensitivity ( $\geq 90 \%$ ), as shown above, this often results in loss of specificity. In contrast, DNA markers tend to have high specificity, but sensitivities much lower than $90 \%$. The authors have developed a method for combining binary marker results (as in the case of DNA markers) with quantitative markers (as in the case of protein markers) to maximize sensitivity and NPV in a population of patients undergoing monitoring for bladder cancer recurrence. The combination of DNA markers with protein markers effectively cherry-picks the population of cancers such that when a protein marker is applied, higher cutoffs can be applied, thus maximizing specificity (Figure 2).

\section{Real time FGFR3 mutation detection in patient urine samples}

This report uses LNA technology and real-time PCR for assessing the mutation status of FGFR 3 in urine samples from patients undergoing recurrence monitoring. Primary amplifications of exons 7, 10, and 15 were performed as described above. An additional quantitative PCR reaction to a reference gene was also performed to determine the amount of amplifiable DNA in each sample. Given the high likelihood of 

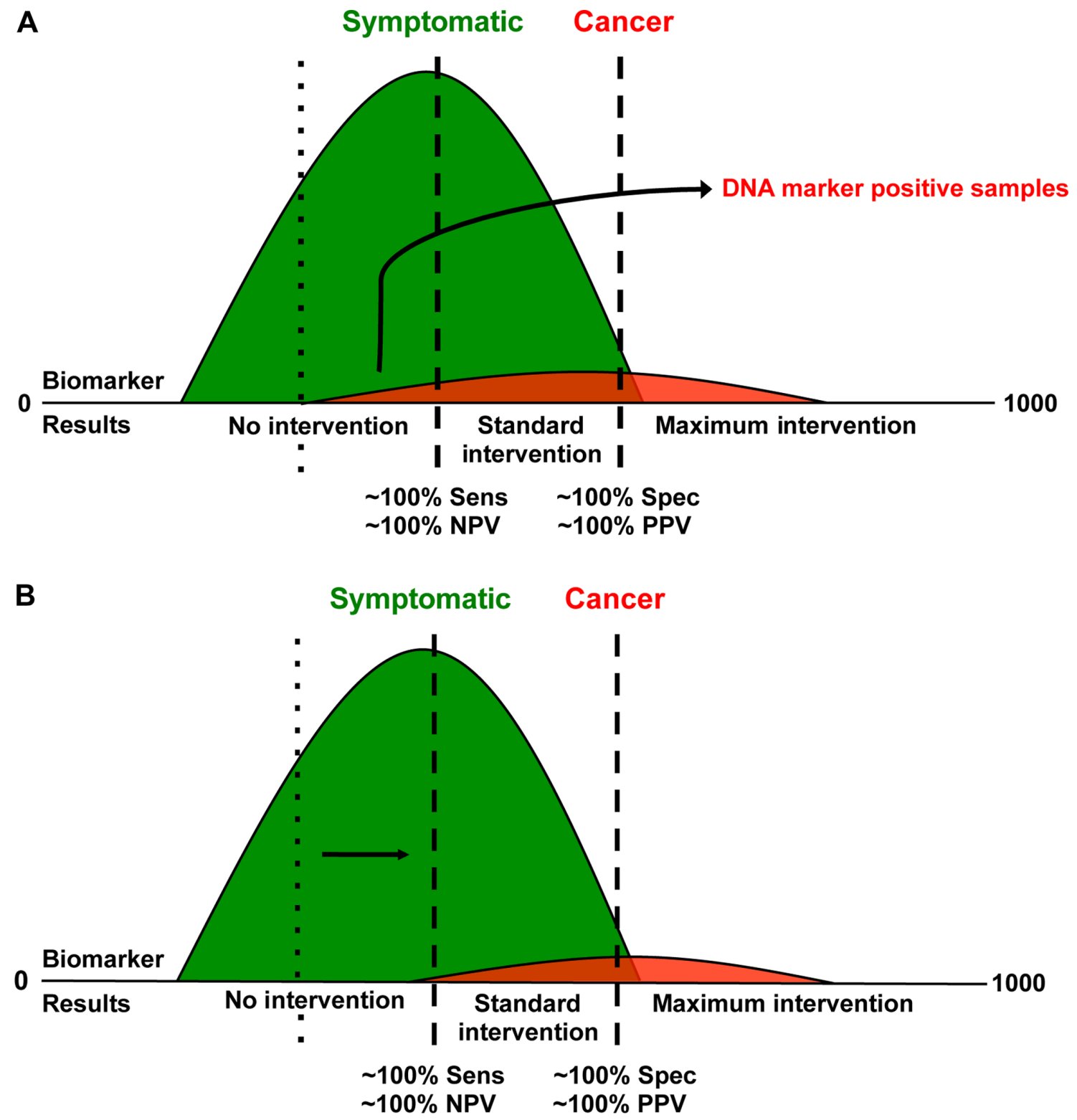

Figure 2 Multi-analyte diagnostic readout: addition of DNA markers reduces population overlap and increases performance. (A) Given the very high specificity of DNA markers, any DNA marker-positive samples can be moved to the high-risk group. (B) This shift essentially depletes the cancer population, allowing higher protein marker cutoffs to be set, increasing specificity while retaining final assay sensitivity $\geq 90 \%$.

Abbreviations: PPV, positive predictive value; NPV, negative predictive value.

DNA degradation that could occur in the urine environment, this step was critical in determining that a minimum DNA input for each sample was used for mutation detection. Out of 323 samples tested, seven did not have enough DNA to reliably obtain an FGFR3 result. These samples were tagged as "inconclusive" and were removed from the final data analysis. Mutation detection was performed on the remaining 316 samples using PCR-clamping with LNAs to block wild-type sequences. LNA is a nucleic acid analogue that enhances hybridization affinity towards complementary DNA and RNA. ${ }^{24}$ Several studies have applied LNA to nucleic acid diagnostics, including the unambiguous detection of single-nucleotide polymorphisms. ${ }^{25,26}$

Real-time PCR amplifications were carried out using the primary PCR products from each patient sample. Each sample was amplified in duplicate in the presence and absence of LNA blocking oligonucleotide (green lines, Figure 3). A representative real-time PCR amplification of a patient sample positive for an FGFR3 mutation in exons 7 is shown in Figure 3, top panel. The bottom trace depicts a single patient sample that is negative for FGFR3 mutations in exon 7. In addition, control urine DNA (blue lines), 

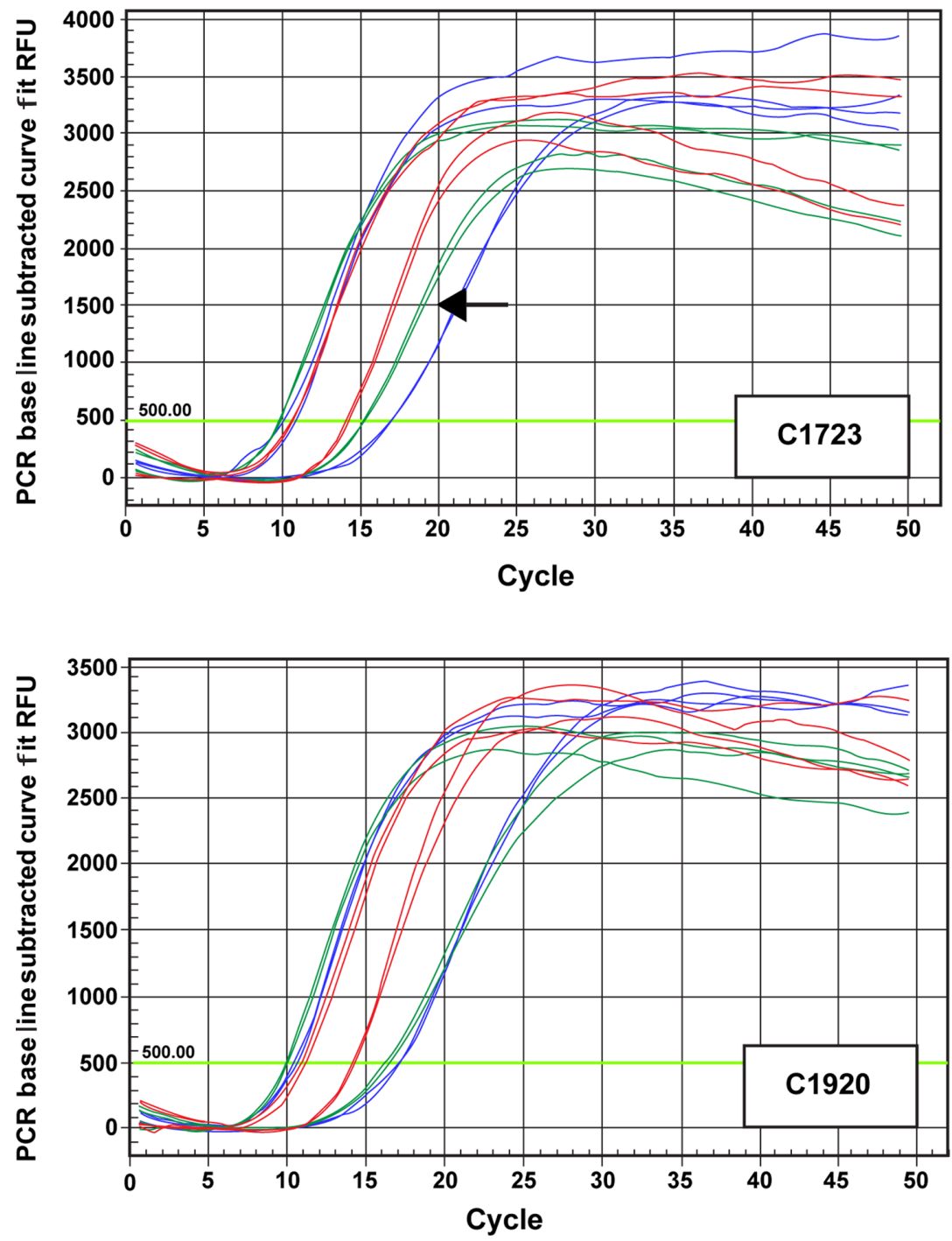

Figure 3 FGFR3 mutation detection in the urine.

Notes: DNA was extracted from the urine of patients undergoing recurrence monitoring and tested for the presence of FGFR3 mutations. Representative samples traces are shown above for a sample that was positive for an exon 7 mutation $(\mathrm{Cl} 723)$ and one that was negative for an exon 7 mutation $(\mathrm{Cl}$ 920). Each sample was amplified in the presence or absence of LNA blocking oligonucleotides (green lines) and run in duplicate. When a mutation is present, amplification curves are shifted to the left (arrow). Control DNA (blue lines) and control DNA with $1 \%$ mutant plasmid (red lines) were also amplified with and without LNA blocking oligonucleotides.

Abbreviations: PCR, polymerase chain reaction; RFU, relative fluorescence units.

and control DNA with $1 \%$ mutant plasmid (red lines) were amplified with and without LNA blocking oligonucleotide. In the top panel, amplification of the patient sample (green) displays a shift to the left (arrow), similar to that seen with the $1 \%$ mutant plasmid controls (red), demonstrating the presence of a mutation. In the bottom panel, the patient sample (green) amplifies similarly to the control urine DNA (blue), suggesting that no mutation is present in this sample. In this sample cohort, 15 mutations were detected, five in the urine of cancer patients, resulting in $10 \%$ sensitivity with $96 \%$ specificity. To verify these results, the mutations detected in the urine of cancer patients were confirmed by direct amplicon sequencing.

\section{Twist I/Nid2 methylation detection}

The Twist1/Nid2 methylation detection assay presented here utilizes DNA extracted from $8 \mathrm{~mL}$ of whole urine. Methylation status was determined using conventional methylation specific PCR for both Twist1 and Nid2. In these assays, 1\% methylated DNA can be reliably detected in as little as $10 \mathrm{ng}$ of converted DNA. To determine that a minimum of $10 \mathrm{ng}$ of converted DNA was assayed for each reaction, bisulfite converted DNA was quantitated using real-time amplification of Actin-B.

Since the bisulfite conversion reaction has a maximum input of 2 ug of DNA, real-time PCR was used prior to conversion to determine total yield from urine. A maximum 
of 2 ug per sample was added to the conversion reaction. Twist1 and Nid2 methylation-specific PCR products were detected at $\sim 120$ and $\sim 140 \mathrm{bp}$, respectively. Unmethylated DNA was used as a negative control, while $1 \%$ methylated DNA mix was used as a positive control in each reaction. Representative gels are shown in Figure 4. Methylation thresholds for Twist1 and Nid2 were established by densitometry. The cutoffs derived from these analyses were used to determine positive samples. At the established cutoffs, Twist 1 methylation was detected in 36 cancer samples and 82 controls, resulting in $75 \%$ sensitivity with $69 \%$ specificity. Nid 2 methylation was detected in 22 cancer samples and 26 controls, resulting in $46 \%$ sensitivity with $90 \%$ specificity.

\section{MADR: combining protein and DNA markers}

Using the MADR approach, threshold and cutoff marker levels were set so that individual marker performance is inconsequential, but that in combination, maximum clinical performance is achieved. For example, although individually FGFR3 and Nid2 sensitivities are low, specificity for both markers was high. No single DNA marker achieved $\geq 90 \%$ sensitivity, but since these markers did not overlap, in combination they increase sensitivity without much loss of specificity. In this fashion, to obtain maximum NPV at high sensitivity, the authors first applied binary markers, and then established cutoffs for the other markers. In combination, patients negative for all markers at the established cutoffs could be excluded from further intervention (power of exclusion). Given that the prevalence of cancer in any given population is low, high NPV may be achieved even with low sensitivity. The CIDD approach requires high sensitivity to add confidence that few, if any, cancers would be missed. The addition of highly specific DNA markers increased sensitivity such that MMP-2 cutoff levels could be raised. This effectively maintained high sensitivity while increasing specificity (Table 3 ).

In this recurrence monitoring population, the combination of all four markers as described above resulted in $97.4 \%$ NPV at a sensitivity of $92 \%$ and the possibility of excluding $51 \%$ of patients who do not have cancer from receiving further tests (Table 3 ). Importantly, the three false negative samples observed here were all of low stage and
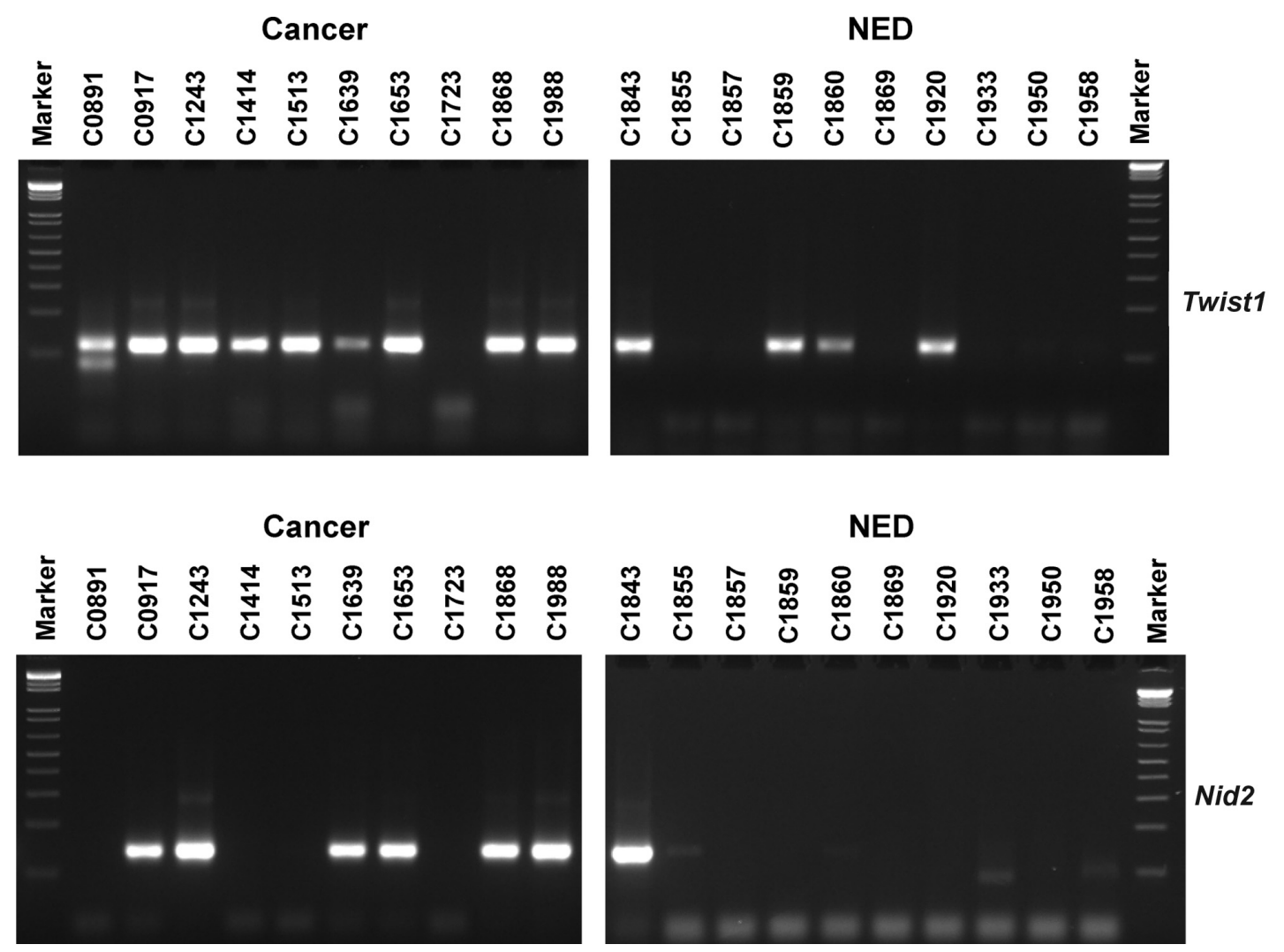

Figure 4 Twist l/Nid2 methylation detection in urine.

Notes: DNA was extracted from $8 \mathrm{~mL}$ of urine and processed to determine methylation of the Twist $/$ and Nid2 genes using methylation-specific polymerase chain reaction. Methylation thresholds were established using densitometry. Representative gels for Twistl and Nid2 are shown above.

Abbreviation: NED, no evidence of disease. 
Table 3 MADR results in maximum clinical performance

\begin{tabular}{|c|c|c|c|c|}
\hline Markers & Cutoffs & NPV* & Sensitivity & Power of exclusion \\
\hline \multirow[t]{3}{*}{ MMP-2 } & MMP-2 $<0.309 \mathrm{ng} / \mathrm{mL}$ & $91.7 \%$ & $90 \%$ & $19 \%$ \\
\hline & & & $(43 / 48)$ & $(51 / 268)$ \\
\hline & & & [77\%-97\%] & [15\%-24\%] \\
\hline \multirow[t]{3}{*}{ MMP-2 + Nid2 } & MMP-2 $<0.418 \mathrm{ng} / \mathrm{mL}$ & $93.6 \%$ & $90 \%$ & $26 \%$ \\
\hline & $\mathrm{Nid} 2<600 \mathrm{k}$ & & $(43 / 48)$ & $(71 / 268)$ \\
\hline & & & [77\%-97\%] & {$[21 \%-32 \%]$} \\
\hline \multirow[t]{3}{*}{ MMP-2 + Nid2 + FGFR3 } & MMP-2 $<0.456 \mathrm{ng} / \mathrm{mL}$ & $94.3 \%$ & $90 \%$ & $29 \%$ \\
\hline & $\mathrm{Nid} 2<600 \mathrm{k}$ & & $(43 / 48)$ & $(77 / 268)$ \\
\hline & & & [77\%-97\%] & {$[23 \%-35 \%]$} \\
\hline \multirow[t]{3}{*}{ MMP-2 + Nid2 + FGFR3 + Twist I } & MMP- $2<1.100 \mathrm{ng} / \mathrm{mL}$ & $97.4 \%$ & $92 \%$ & $51 \%$ \\
\hline & $\mathrm{Nid} 2<600 \mathrm{k}$ & & $(44 / 48)$ & $(136 / 268)$ \\
\hline & Twistl $<249 \mathrm{k}$ & & [80\%-98\%] & [44\%-57\%] \\
\hline
\end{tabular}

Notes: Urine samples from 48 individuals with bladder cancer and 268 NED patients were tested for MMP-2, FGFR3, Twist I, and Nid2. High specificity of the DNA markers allowed for higher MMP-2 cutoffs to be set as each DNA marker was added, increasing specificity and NPV. Although individually low, in combination the four markers results in $92 \%$ sensitivity with the power to exclude $51 \%$ of cancer-free patients from further intervention with very high confidence ( $97.4 \%$ NPV).

Abbreviations: MMP, matrix metalloproteinases; NPV, negative prediction value; NED, no evidence of disease.

grade (TaG1). At 92\% sensitivity, of all patients (cancers and controls) being monitored at any given time, $43 \%$ would be triaged from receiving further tests.

\section{Discussion}

This study describes the development of a noninvasive diagnostic test for the triaging of patients being evaluated for potential bladder cancer recurrence. The assay presented here combines the sensitivity of protein markers with the specificity of DNA markers for optimum clinical performance. Here, MMP-2 protein levels are coupled with methylation analysis of the Twist1 and Nid2 genes and mutational analysis of the FGFR3 gene. Using this approach, $51 \%$ of patients being monitored for bladder cancer recurrence, but who do not have cancer, could have been excluded from further invasive intervention with very high confidence (97\% NPV) (Table 3). The MADR approach builds on the CIDD concept the authors have previously described. ${ }^{12}$ Using CIDD, patients would be stratified into three groups: one that is cancer free and could be excluded from undergoing further evaluation; a second that simply receives the already scheduled standard of care; and a third that has a high likelihood of cancer and could receive accelerated intervention.

FGFR3 mutations are prevalent in noninvasive, low-grade bladder tumors. ${ }^{15-18}$ Although sensitivity for FGFR3 alone was low in this sample set (11\%), the presence of an FGFR3 mutation is indicative of a high likelihood of cancer. Given the high specificity of FGFR3 mutations to bladder cancer, FGFR3 mutations in urine could be used to establish a group of patients who are at high risk and who could benefit from accelerated intervention. In addition, studies have further shown that patients who had a negative cystoscopy but a positive
FGFR3 mutation in urine at any one monitoring interval are often have recurrent cancer in subsequent visits. ${ }^{27}$

The authors have previously demonstrated how MMPs can be used to separate bladder cancer patients from cancer-free controls, as depicted in Figure 1A. Although the specificity in these studies was high, specificity drops in high-risk populations as MMP levels converge. Given the potential high specificity of DNA markers, the addition of these markers to the existing protein marker distribution essentially depletes the cancers from the population to which the protein marker is applied (Figure 2A). Since the number of cancers that overlap with the cancer-free individuals is reduced, the protein marker cutoff can then be shifted, resulting in increased specificity (Figure 2B). As the assay continues to be improved, other validated bladder cancer markers with high specificity could be added and others removed to continue to increase performance with a minimum number of markers. In the study presented here, the application of MADR using these bladder cancer markers in patients undergoing recurrence monitoring establishes new threshold levels that provide information as to which patients might be excluded from invasive procedures at the given monitoring interval and those who might benefit from accelerated intervention. Traditionally, protein and DNA markers are multiplexed as independent marker sets but not combined into a protein and DNA multiplex format. Although protein markers can achieve high sensitivity due to their quantitative nature, they often fall short of the specificity required to add clinical utility. Therefore, multiplexing of a significant number of protein markers (eg, protein-based expression patterns) are used to increase specificity. In contrast, independent DNA alterations tend to have high specificity, but require significant multiplexing to achieve high sensitivity ( $\geq 90 \%)$. 
The current study demonstrates that the unique combination of DNA and protein markers into one assay improved clinical performance with a minimal number of markers resulting in lower assay complexity. The resulting diagnostic assay is the first to combine the best performance characteristics of protein biomarkers and DNA biomarkers into one assay for optimal clinical performance.

\section{Acknowledgments}

L Anderson, K Johnson, and J Alexander assisted with manuscript preparation. MA Moses provided clinical and study advice. The authors dedicate this work to the memory of Dr Ian C Summerhayes, their dear friend and colleague.

\section{Disclosure}

In relation to this work, RJ Karnes, A Feldman, and E Zwarthoff have nothing to disclose; C Fernandez, J Millholland, and AP Shuber are employees of Predictive Biosciences.

\section{References}

1. American Cancer Society: Cancer Facts and Figures. Available from: www.cancer.org. Accessed March 6, 2008.

2. Botteman MF, Pashos CL, Redaelli A, Laskin B, Hauser R. The health economics of bladder cancer: a comprehensive review of the published literature. Pharmacoeconomics. 2003;21(18):1315-1330.

3. Hong YM, Loughlin KR. Economic impact of tumor markers in bladder cancer surveillance. Urology. 2008;71(1):131-135.

4. Duggan B, Williamson K. Molecular markers for predicting recurrence, progression and outcomes of bladder cancer (do the poster boys need new posters?). Curr Opin Urol. 2004;14(5):277-286.

5. Kleiner DE, Stetler-Stevenson WG. Matrix metalloproteinases and metastasis. Cancer Chemother Pharmacol. 1999;43 Suppl:S42-S51.

6. Moses MA. The regulation of neovascularization of matrix metalloproteinases and their inhibitors. Stem Cells. 1997;15(3):180-189.

7. Eissa S, Ali-Labib R, Swellam M, Bassiony M, Tash F, El-Zayat TM. Noninvasive diagnosis of bladder cancer by detection of matrix metalloproteinases (MMP-2 and MMP-9) and their inhibitor (TIMP-2) in urine. Eur Urol. 2007;52(5):1388-1396.

8. Fernandez CA, Yan L, Louis G, Yang J, Kutok JL, Moses MA. The matrix metalloproteinase-9/neutrophil gelatinase-associated lipocalin complex plays a role in breast tumor growth and is present in the urine of breast cancer patients. Clin Cancer Res. 2005;11(15): 5390-5395.

9. Moses MA, Wiederschain D, Loughlin KR, Zurakowski D, Lamb CC, Freeman MR. Increased incidence of matrix metalloproteinases in urine of cancer patients. Cancer Res. 1998;58(7):1395-1399.

10. Smith ER, Manfredi M, Scott RM, Black PM, Moses MA. A recurrent craniopharyngioma illustrates the potential usefulness of urinary matrix metalloproteinases as noninvasive biomarkers: case report. Neurosurgery. 2007;60(6):E1148-E1149; discussion E1149.

Research and Reports in Urology

\section{Publish your work in this journal}

Research and Reports in Urology is an international, peer-reviewed, open access journal publishing original research, reports, editorials, reviews and commentaries on all aspects of adult and pediatric urology in the clinic and laboratory including the following topics: Pathology, pathophysiology of urological disease; Investigation and treatment of
11. Smith ER, Zurakowski D, Saad A, Scott RM, Moses MA. Urinary biomarkers predict brain tumor presence and response to therapy. Clin Cancer Res. 2008;14(8):2378-2386.

12. Fernandez CA, Wszolek MF, Loughlin KR, Libertino JA, Summerhayes IC, Shuber AP. A novel approach to using matrix metalloproteinases for bladder cancer. $J$ Urol. 2009;182(5):2188-2194.

13. Cappellen D, De Oliveira C, Ricol D, et al. Frequent activating mutations of FGFR3 in human bladder and cervix carcinomas. Nat Genet. 1999;23(1):18-20.

14. Johnson DE, Williams LT. Structural and functional diversity in the FGF receptor multigene family. Adv Cancer Res. 1993;60:1-41.

15. Tomlinson DC, Baldo O, Harnden P, Knowles MA. FGFR3 protein expression and its relationship to mutation status and prognostic variables in bladder cancer. J Pathol. 2007;213(1):91-98.

16. Billerey C, Chopin D, Aubriot-Lorton MH, et al. Frequent FGFR3 mutations in papillary non-invasive bladder (pTa) tumors. Am J Pathol. 2001;158(6):1955-1959.

17. Junker K, van Oers JM, Zwarthoff EC, Kania I, Schubert J, Hartmann A. Fibroblast growth factor receptor 3 mutations in bladder tumors correlate with low frequency of chromosome alterations. Neoplasia. 2008;10(1):1-7.

18. van Oers JM, Zwarthoff EC, Rehman I, et al. FGFR3 mutations indicate better survival in invasive upper urinary tract and bladder tumours. Eur Urol. 2009;55(3):650-657.

19. Gort EH, Suijkerbuijk KP, Roothaan SM, et al. Methylation of the TWIST1 promoter, TWIST1 mRNA levels, and immunohistochemical expression of TWIST1 in breast cancer. Cancer Epidemiol Biomarkers Prev. 2008;17(12):3325-3330.

20. Okada T, Suehiro Y, Ueno K, et al. TWIST1 hypermethylation is observed frequently in colorectal tumors and its overexpression is associated with unfavorable outcomes in patients with colorectal cancer. Genes Chromosomes Cancer. 2010;49(5):452-462.

21. Ulazzi L, Sabbioni S, Miotto E, et al. Nidogen 1 and 2 gene promoters are aberrantly methylated in human gastrointestinal cancer. Mol Cancer. 2007;6:17.

22. Xie F, Li K, Ouyang X. Twist, an independent prognostic marker for predicting distant metastasis and survival rates of esophageal squamous cell carcinoma patients. Clin Exp Metastasis. 2009;26(8): $1025-1032$.

23. Renard I, Joniau S, van Cleynenbreugel B, et al. Identification and validation of the methylated TWIST1 and NID2 genes through real-time methylation-specific polymerase chain reaction assays for the noninvasive detection of primary bladder cancer in urine samples. Eur Urol. 2010;58(1):96-104.

24. Petersen M, Wengel J. LNA: a versatile tool for therapeutics and genomics. Trends Biotechnol. 2003;21(2):74-81.

25. Bruge F, Littarru GP, Silvestrini L, Mancuso T, Tiano L. A novel Real Time PCR strategy to detect SOD3 SNP using LNA probes. Mutat Res. 2009;669(1-2):80-84.

26. Orum H, Jakobsen MH, Koch T, Vuust J, Borre MB. Detection of the factor $\mathrm{V}$ Leiden mutation by direct allele-specific hybridization of PCR amplicons to photoimmobilized locked nucleic acids. Clin Chem. 1999;45(11):1898-1905.

27. Zuiverloon TC, van der Aa MN, van der Kwast TH, et al. Fibroblast growth factor receptor 3 mutation analysis on voided urine for surveillance of patients with low-grade non-muscle-invasive bladder cancer. Clin Cancer Res. 2010;16(11):3011-3018.

\section{Dovepress}

urological disease; Pharmacology of drugs used for the treatment of urological disease. The manuscript management system is completely online and includes a very quick and fair peer-review system, which is all easy to use. Visit http://www.dovepress.com/testimonials.php to read real quotes from published authors. 